\title{
Detection of bacteriuria in women attending local authority antenatal clinics using the dip-inoculum transport medium spoon method
}

\author{
C. S. GOODWIN AND AUDREY E. STEWART \\ From the Public Health Laboratory and the Public Health Department, Portsmouth
}

SYNOPSIS Four hundred and seventy-eight pregnant women attending local authority clinics in Portsmouth were screened for bacteriuria using the dip-inoculum transport medium (DITM) spoon method. The detection of asymptomatic bacteriuria in $5 \%$ of the patients with a very low proportion of equivocal results $(0.83 \%)$ suggests that this is an efficient method; and large numbers of urine specimens could be sampled with relatively little work for the laboratory. A cystineMacConkey medium was devised for incorporation in the spoons. General practitioners treated the women with bacteriuria, and remained responsible for home delivery and postpartum examinations. The possibility of successful cooperation between non-hospital clinics, a bacteriological laboratory, and general practitioners is demonstrated.

The proportion of overt urinary tract infections in pregnant women that are preceded by asymptomatic bacteriuria has been reported to be between $60 \%$ (Kass, 1960; Turner, 1961) and 30\% (Dixon and Brant, 1967). Detection of such bacteriuria not only allows the prevention of many overt infections, but may also indicate women who are candidates for chronic bacteriuria and subsequent chronic pyelonephritis (Garrod, Shooter, and Curwen, 1954; Pinkerton, Wood, Williams, and Calman, 1961; Brumfitt and Percival, 1964), or hypertension (Freedman, Phair, Seki, Hamilton, and Nefzger, 1965). An essential prerequisite for satisfactory bacteriological analysis of urines is the ability to obtain a very low proportion of contaminated specimens. This is difficult to achieve even in a hospital environment, and in this study necessitated the attention of a midwife for four minutes for each patient. However, the demonstration of the possibility of efficient cooperation between a local authority antenatal maternity service, a bacteriological laboratory, and general practitioners could lead to the application of existing knowledge concerning bacteriuria of pregnancy to the nonhospital population. Busy bacteriological laboratories are unlikely to be able to process large numbers of additional urine specimens in a conventional way, but the dip-inoculum transport Received for publication 3 November 1967. medium (DITM) 'spoon' offers a solution to the problem (Mackey and Sandys, 1965). The DITM spoon avoids the need to refrigerate and transport large numbers of urine specimens, $95 \%$ of which from pregnant women would not contain a significant number of bacteria. Because the urine is sampled immediately after passing, multiplication of contaminants during delayed transit from outlying clinics is avoided.

\section{WOMEN AND METHODS}

At three antenatal clinics of the Portsmouth local authority maternity service, all patients attending between 1 February and 30 June 1967 for the first booking of their pregnancy were included in this study. In Portsmouth a woman requesting home delivery may be referred by her general practitioner to a local authority clinic for antenatal care. In the clinic a midwife instructed each patient in the technique of obtaining an uncontaminated midstream specimen of urine. Vulval cleansing and separation of the labia during micturition reduce the frequency of contamination of specimens and equivocal results (Wood, Pinkerton, and Calman, 1960 ; Little, 1966); and for vulval cleansing sterile water achieves as satisfactory results as chlorhexidine, without the disadvantage of reducing the count of bacteria in the specimen (Roberts, Robinson, and Beard, 1967). After washing the hands, two cotton wool swabs moistened with tap water were used for swabbing the vulva, and the very low percentage of colony counts 
in the equivocal range of $10^{4}$ to $10^{5}$ organisms $/ \mathrm{ml}$ obtained in this study vindicated this simple procedure. One midwife during a 60 -minute clinic could personally supervise the collection of urine specimens from not more than 12 patients. A midstream urine specimen was caught in a disposable sterile aluminium foil bowl, 5 in. in diameter and $2 \frac{1}{2}$ in. deep.

The polypropylene DITM spoon (Medical Wire and Equipment Co., Bath Ltd.), in which was incorporated cystine-MacConkey agar, was dipped vertically into the urine so that the medium was covered, and the spoon then returned to its own sterile universal jar, with moistened cotton wool in the bottom. The rim of the foil bowl could be pinched to allow accurate pouring into a sterile honey jar. A smaller polypropylene pot (Brumfitt and Percival, 1964) is a satisfactory alternative both for collection and for transport. The cystineMacConkey medium was made by the addition of $8 \mathrm{ml}$ of a $1.6 \%$ solution of $\mathrm{L}$-cystine in normal $\mathrm{NaOH}$ to a litre of 'Oxoid MacConkey agar no. 2', the $\mathrm{pH}$ remaining at $7 \cdot 3$. The reasons for using this medium are discussed below. The DITM spoon is easily autoclaved and used again.

From the first 50 patients both DITM spoons and midstream urine specimens were examined to determine whether the DITM spoon accurately reflected colony counts on agar plates. This was found to be so (see Results), and for subsequent screening the only specimen received from each new patient was a DITM spoon in its bottle. From a patient found to have possibly significant bacteriuria, ie, greater than $10^{4}$ organisms $/ \mathrm{ml}$ (Leigh and Williams, 1964), a repeat DITM spoon was requested with an accompanying midstream urine specimen which was plated fully on agar plates, the pathogen identified as being the same organism as originally isolated and sensitivity tests performed. A centrifuged deposit of the urine specimen was examined. The laboratory findings were reported to the general practitioner, with a request to initiate appropriate chemotherapy. The urine was examined one week after the cessation of chemotherapy and also one month after delivery to assess the need for further chemotherapy and possibly hospital investigation.

\section{RESULTS}

Of 478 pregnant women examined at their first booking, 24 patients were found to have a significant bacteriuria of greater than $10^{5}$ organisms $/ \mathrm{ml}$ of $\overrightarrow{2}$ urine in two consecutive midstream specimens, $\frac{}{-}$ giving a rate of asymptomatic bacteriuria of $5 \% \cdot \overrightarrow{\vec{F}}$ The volume of urine sampled by the DITM spoon was found to be $0.002 \mathrm{ml}$, which is similar to that reported by Mackey and Sandys (1965). These $\frac{\bar{D}}{\bar{D}}$ authors also delineated the different 'conversion $\overparen{\mathbb{D}}$ factors' for Gram-negative bacilli and Gram-positive 을 cocci that are required to calculate colony counts, $\infty$ and these factors were confirmed in the present $\vec{\circ}$ study. Thus on a DITM spoon 200 colonies of $\overrightarrow{\vec{H}}$ Gram-negative bacilli were found to be equivalent $\vec{\omega}$ to $10^{5}$ organisms $/ \mathrm{ml}$ of urine, while 400 colonies of Gram-positive cocci on the DITM spoon were equivalent to $10^{5}$ organisms per millilitre of urine.

In the first 50 specimens the number of colonies on the DITM spoon paralleled the density of $\frac{\vec{A}}{\mathrm{f}}$ bacteria determined by conventional plating (Table I). In Table I the plate counts for the groups $\mathrm{C}, \underset{ }{\rightarrow}$ $D, E$, and $F$ would be expected to be slightly higher $C$ than the DITM spoon count because a period of time was required for transport of specimens to the $\vec{\varphi}$ laboratory.

In four out of 478 patients $(0.83 \%)$ an 'equivocal count' of $10^{4}$ to $10^{5}$ organisms $/ \mathrm{ml}$ was obtained. The second specimen of urine from each of these four patients was found to contain less than $10^{3} \overline{\bar{\partial}}$ organisms/ml with no colonies on the DITM spoon. In three of these patients the equivocal $\triangle$ count involved Gram-positive cocci and the fourth $\overrightarrow{\bar{O}}$ involved Gram-negative bacilli. In 18 out of the $\overline{3}$ other 474 specimens the spoon colony count indicated between $10^{3}$ and $10^{4}$ organisms $/ \mathrm{ml}$ of urine, and in nearly all instances these were Gram-음 positive cocci. In the other 456 DITM specimens? there were either no colonies on the spoon or a 3

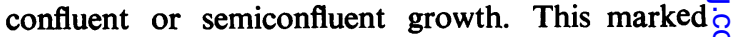
difference between significant and non-significant $\frac{3}{3}$ bacteriurias was also reported by Mackey and Sandys (1965).

In the centrifuged deposit of the urine specimens $\frac{D}{O}$

TABLE I

COMPARATIVE COLONY COUNTS OF URINE SPECIMENS USING THE DITM SPOON AND AGAR PLATES

\begin{tabular}{lclcc} 
Group & No. of Specimens & $\begin{array}{l}\text { Colonies on DITM } \\
\text { Spoon }\end{array}$ & $\begin{array}{l}\text { Organisms/ml of Urine Calculated } \\
\text { from Spoon Colonies }\end{array}$ \\
\hline A & 37 & 0 & $<10^{3}$ & $\begin{array}{l}\text { Plate Count } \\
\text { (organisms/ml) }\end{array}$ \\
B & 3 & 2 & $1 \times 10^{3}$ & $1 \times 10^{3}$ \\
C & 1 & 8 & $4 \times 10^{3}$ & $6 \times 10^{3}$ \\
D & 4 & 20 & $1 \times 10^{4}$ & $2 \times 10^{4}$ \\
E & 1 & $40^{1}$ & $1 \times 10^{4}$ & $3 \times 10^{4}$ \\
F & 1 & $60^{1}$ & $1 \cdot 5 \times 10^{4}$ & $2 \times 10^{4}$ \\
G & 3 & Confluent & $>10^{5}$ & $>10^{5}$
\end{tabular}

${ }^{1}$ Gram-positive cocci 
of the 24 bacteriuric women the number of leucocytes per high-power field varied widely. In 17 specimens there were between 1 and 6 leucocytes per high-power field, in four specimens between 8 and 20 leucocytes, and in three specimens there were more than 30 leucocytes per high-power field.

The organisms identified in the 24 bacteriuric patients were Escherichia coli in 18 patients, Proteus mirabilis in four patients, Klebsiella aerogenes in one patient, and Staphylococcus albus in one patient.

Symptoms of acute pyelitis had developed in three women by the time the second urine specimen was obtained. In one of these women the causative organism was Staph. albus. The isolate of $K$. aerogenes was sensitive in vitro to nitrofurantoin, but treatment with this drug alone failed to eradicate the infection. This failure was possibly due to the urease action of the Klebsiella rendering the urine alkaline, nitrofurantoin being ineffective in an alkaline urine (Barber and Garrod, 1963). Concomitant treatment with nitrofurantoin and ascorbic acid $100 \mathrm{mg}$ tds to render the urine acid resulted in eliminating this infection.

\section{DISCUSSION}

The incidence of asymptomatic bacteriuria in pregnant women has been found to be between $4.7 \%$ and $7 \%$ (Brumfitt, Davies, and Rosser, 1961; Little, 1966), and any simplified method for screening pregnant women, to be considered satisfactory, must achieve a similar detection rate. In this small series, $5 \%$ of 478 women were found to have significant bacteriuria, which suggests that the technique and the medium were satisfactory. The low percentage of equivocal results of $0.83 \%$ indicates that the collection technique of the midstream specimens was efficient (Brumfitt, 1965). The women discovered to have asymptomatic bacteriuria contrasted with others complaining of cystitis-like symptoms but who were found not to have a urinary tract infection. Symptoms such as dysuria and frequency are common in women, and in many cases a significant bacteriuria is not found (Gallagher, Montgomerie, and North, 1965).

For large-scale screening for bacteriuria the choice may be between the DITM spoon technique and the absorbent paper inoculum of Leigh and Williams (1964). Although the latter method probably entails less preparation of suitable media, the DITM spoon offers the advantages, in outlying clinics without refrigerators, of a longer shelf life and a safer transport vehicle than agar plates. With increasing interest in initiating screening programmes a reliable transport vehicle to and from clinics is essential; and, in addition, the spoon sampling technique offers less opportunity for administrative error and contamination of inoculum sites than multiple inoculated plates.

When one medium alone is used for screening for bacteriuria the choice is determined by its ability to support the growth of urinary tract pathogens, and prevent the swarming of Proteus species. The cystine, lactose, electrolyte deficient medium with bromthymol blue, suggested for the DITM spoon (Mackey and Sandys, 1966), causes unfamiliar colonial appearances of common organisms, and allows the growth of diphtheroids and other nonpathogens; but the incorporation of cystine is of value because cystine-dependent $E$. coli (Gillespie, 1952) and Salmonella typhi (Jacobson, 1910) grow to a normal size. MacConkey agar (Oxoid 'without salt') is used for screening for bacteriuria (Leigh and Williams, 1964), but the concentration of neutral red, $0.075 \mathrm{~g} /$ litre, is inhibitory to some strains of $E$. coli. MacConkey agar (Oxoid no. 2) with neutral red $0.05 \mathrm{~g} /$ litre rarely supports the growth of Staphylococcus aureus and poorly supports some strains of Staph. albus and Streptococcus faecalis. However, the addition of cystine improves the growth of Staph. albus and Strep. faecalis and also supports group $\mathbf{B}$ and group $\mathbf{C}$ haemolytic streptococci, including a group B strain obtained by suprapubic bladder aspiration in a girl with pyelonephritis (Goodwin, unpublished data). Using a MacConkey medium, airborne contaminants are not a problem.

If the cystine, lactose, electrolyte-deficient medium is used in the DITM spoons, to equate the two specimens needed for confirmation of bacteriuria the fuller investigation of the second specimen would need to involve plating on cystine, lactose, electrolyte-deficient agar. Preparation of an additional medium for routine use might be unwelcome, but the cystine-MacConkey medium, described here, is now in this laboratory the only MacConkey medium prepared for routine work.

Urinary leucocyte counts in the 24 women with bacteriuria were found in 17 women to be within the normal range for a pregnant woman. It has frequently been reported that a significant bacteriuria can exist despite a normal leucocyte count (Loudon and Greenhalgh, 1962; Brumfitt, 1965).

The successful cooperation obtained between non-hospital clinics, a bacteriological laboratory, and general practitioners was very encouraging and may be a reproducible pattern.

The authors thank Dr D. J. H. Payne, the Director of the Public Health Laboratory, Dr D. A. Skan, and Dr P. G. Roads, the Medical Officer of Health, Portsmouth, for their advice and encouragement. 


\section{REFERENCES}

Barber, M., and Garrod, L. P. (1963). Antibiotic and Chemotherapy p. 289. Livingstone, Edinburgh.

Brumfitt, W. (1965). J. clin. Path., 18, 550.

_- Davies, B. I., and Rosser, E. (ap) I. (1961). Lancet, 2, 1059.

, and Percival, A. (1964). J. clin. Path., 17, 482.

Dixon, H. G., and Brant, H. A. (1967). Lancet, 1, 19.

Freedman, L. R., Phair, J. P., Seki, M., Hamilton, H. B., and Nefzger, M.D. (1965). Yale J. Biol. Med., 37, 262.

Gallagher, D. J. A., Montgomerie, J. Z., and North, J. D. K. (1965). Brit. med. J., 1, 622 .

Garrod, L. P. Shooter, R. A., and Curwen, M.P. (1954). Ibid., 2, 1003.
Gillespie, W. A. (1952), J. Path. Bact., 64, 551.

Jacobson, K. A. (1910). Zbl. Bakt., I. Abt. Orig., 56, 208.

Kass, E. H. (1960). Bact. Rev., 24, 177.

Leigh, D. A., and Williams, J. D. (1964). J. clin. Path., 17, 498.

Little, P. J. (1966). Lancet, 2, 925.

Loudon, I. S. L., and Greenhalgh, G. P. (1962). Ibid., 2, 1246.

Mackey, J. P., and Sandys, G. H. (1965). Brit. med. J., 2, 1286. (1966). Ibid., 1, 1173.

Pinkerton, J. H. M., Wood, C., Williams, E. R., and Calman, R. M. (1961). Ibid., 2, 539

Roberts, A. P., Robinson, R. E., and Beard, R. W. (1967). Ibid., 1, 400.

Turner, G. C. (1961). Lancet, 2, 1062.

Wood, E. C., Pinkerton, J. H. M., and Calman, R. M. (1960) Brit. med. J., 1, 961 . 